\title{
Quadratic optomechanical cooling of a cavity-levitated nanosphere
}

\author{
N. P. Bullier $\odot,{ }^{*}$ A. Pontin $\odot,{ }^{\dagger}$ and P. F. Barker ${ }^{\ddagger}$ \\ Department of Physics and Astronomy, University College London, Gower Street, London WC1E 6BT, United Kingdom
}

(Received 30 June 2020; accepted 16 June 2021; published 28 July 2021)

\begin{abstract}
We report on cooling of the center-of-mass motion of a nanoparticle via a coupling between its motion and the optical field within a high finesse cavity. The resulting coupling is purely quadratic in displacement and gives rise to a van der Pol nonlinear damping. These dynamics are analogous to conventional parametric feedback where the cavity provides passive feedback without an additional measurement. We show experimentally that as feedback cooling, the resulting energy distribution is strongly nonthermal and can be controlled by the nonlinear damping induced by the cavity. Our work represents a demonstration of a cavity cooling dominated by this type of coupling across all optomechanical platforms.
\end{abstract}

DOI: 10.1103/PhysRevResearch.3.L032022

The field of cavity optomechanics has made significant progress over the last decade by controlling and tailoring the interaction between an optical field and a mechanical oscillator. Mechanical modes have been brought into the quantum regime $[1,2]$. This includes quantum mechanical squeezing of an oscillator below its zero point fluctuations [3] and entanglement between two mechanical oscillators [4,5]. More recently, the creation of oscillators levitated in vacuum using optical, electrical, or magnetic fields has been realized. These systems offer greater decoupling from the environment, and they typically only have a few low-frequency mechanical degrees of freedom [6-8], but can also introduce new optomechanical degrees of freedom via their rotational motion [9]. Levitated systems offer also the opportunity for exploring thermodynamics at the nanoscale in the underdamped regime $[10,11]$.

There is a general interest in optomechanics to exploiting nonlinearities of the interaction, in particular, those which are quadratic in the oscillator motion. Apart from interesting dynamics [12-16], this type of nonlinear coupling is sought after for its potential applications in the quantum regime [17-19]. However, it is often too small to be relevant and easily overwhelmed by a residual linear coupling. This has led the community to look for viable ways to enhance nonlinear couplings, a few notable examples being avoided crossings in membrane-in-the-middle systems $[20,21]$ and purposefully engineered photonic crystal cavities [22-24].

In this respect, levitated cavity optomechanical systems offer significant advantages. There is great freedom to engi-

\footnotetext{
*Present address: National Physical Laboratory, Hampton Road, Teddington TW11 0LW, United Kingdom.

†a.pontin@ucl.ac.uk

†p.barker@ucl.ac.uk

Published by the American Physical Society under the terms of the Creative Commons Attribution 4.0 International license. Further distribution of this work must maintain attribution to the author(s) and the published article's title, journal citation, and DOI.
}

neer the potential experienced by a levitated nanoparticle to the point where the trapping fields can be switched off to observe free evolution. As for the membrane-in-the-middle setup, control over the oscillator mean position within the cavity standing wave enables an optical coupling that is linear or quadratic in the particle motion [25-27]. However, if the trapping potential is solely given by the cavity field, the particle is naturally attracted by the optical gradient force toward an intensity maximum where the coupling is purely quadratic. This feature has remained largely unexplored in levitated cavity optomechanics since experimental efforts mainly focused on enhancing linear coupling which typically provides a better cooling efficiency.

Here, we demonstrate a coupling quadratic in the particle motion that is strong enough to cool a levitated nanosphere by more than two orders of magnitude. Importantly, the resulting oscillator dynamics is equivalent to that obtained with active parametric feedback often used in optical tweezers [28,29]. However, unlike feedback damping, cavity cooling is passive and does not rely on a position measurement. The cavity fieldparticle interaction can be viewed as the field measuring the particle position since its motion is encoded in the field. This information, however, is not actionable until one measures the field fluctuations. In this second measurement the information on the particle motion is usually degraded for example by quantum efficiency. Finally, we describe the resulting highly nonthermal state of the mechanical motion which compares well with our theoretical description of the experiment.

We create our optomechanical system by levitating a highly charged silica nanosphere in a composite potential obtained by overlapping an electrodynamic potential and an optical standing wave. The former is provided by a linear Paul trap, the latter resulting from a high finesse Fabry-Pérot cavity. In our typical scenario, the nanoparticle is optically trapped along the main axis by the gradient force in one of the cavity antinodes while radial confinement is guaranteed by the Paul trap potential. The presence of the dielectric particle in the cavity field shifts its resonance frequency by $\Delta(x)=-U_{o} \cos ^{2}(k x)$, where $x$ is the particle position along 


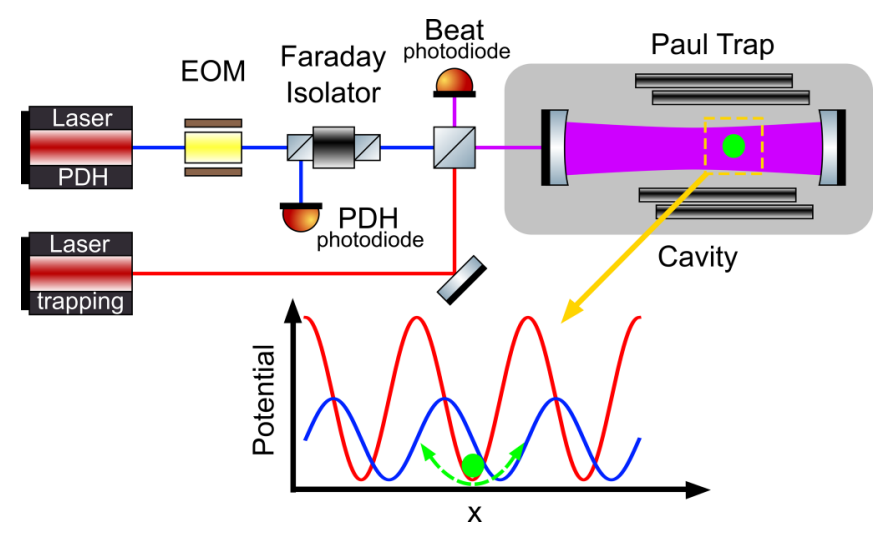

FIG. 1. Simplified layout of the experiment [30]. A weak probe beam is used to lock the cavity by implementing a PDH scheme. A second beam, generated by a different laser, is used to optically trap the particle. By detecting the beat note of the lasers, the trapping beam is offset phase locked to the PDH beam one FSR apart $\sim 10.3 \mathrm{GHz}$. The Paul trap is aligned in the cavity transverse direction but kept far from the cavity center where the probe and trapping beam are out of phase.

the cavity axis, with its origin at the antinode nearest the cavity center, $k=2 \pi / \lambda$ is the optical field wave number, and $U_{o}=\frac{3}{2} \frac{V}{V_{m}} \frac{\epsilon-1}{\epsilon+2} \omega_{l}$ is the maximum frequency shift obtained at intensity antinodes. Here, $V$ and $V_{m}$ are the sphere and cavity mode volume, respectively, $\epsilon$ denotes the nanosphere permittivity, and $\omega_{l}$ the laser frequency. It is clear that $\Delta(x)$ can give rise to a highly nonlinear optomechanical coupling.

A schematic overview of our experimental setup is shown in Fig. 1. Two Nd:YAG lasers with a wavelength of $\lambda \simeq$ $1064 \mathrm{~nm}$ drive a $L_{\text {cav }}=14.58 \pm 0.02 \mathrm{~mm}$ long cavity with a finesse of $\mathcal{F}=36000$ (half linewidth $\kappa / 2 \pi=143 \pm 1 \mathrm{kHz}$, input rate $\kappa_{\text {in }} / 2 \pi=69 \pm 4 \mathrm{kHz}$ ). The cavity has a nearly confocal configuration with a waist of $w_{\mathrm{st}}=62 \mu \mathrm{m}$ (i.e., $\left.V_{m}=\pi w_{\text {st }}^{2} L_{\text {cav }} / 4\right)$. One laser is used as a weak probe field and is locked to the cavity by implementing a Pound-DreverHall (PDH) scheme. The second is used to optically trap the nanoparticle. Its frequency is offset locked to the weak beam by one free spectral range $\left(\mathrm{FSR}=c / 2 L_{\mathrm{cav}}=10.27 \pm\right.$ $0.02 \mathrm{GHz}$ ) away and its detuning from the cavity resonance can be precisely controlled. Both beams are injected in the cavity with a mode matching $>91 \%$.

The particle is charged during the loading process by means of electrospray ionization and captured directly in a medium vacuum within the Paul trap (see Refs. [30-36]). We use commercial silica nanospheres of mass $m=4.88 \pm$ $0.03 \times 10^{-17} \mathrm{~kg}$ [37] and radius $185 \pm 2 \mathrm{~nm}$. The trap is mounted on a three-axis translation stage. This allows us to enhance the linear coupling of the probe field by trapping optically away from the cavity center [38]. Second, it allows us to strongly suppress excess micromotion. Indeed, contrary to previous implementations [25,39], the dynamics along the cavity axis is ideally micromotion free. We can measure and control the position of the optical trapping site referred to the cavity center with a resolution of $\sim 10 \mu \mathrm{m}$, mainly limited by the particle thermal variance before optical confinement.

In the following we focus on the center-of-mass (c.m.) motion along the cavity axis. The nonlinear dynamical equation of motion for the oscillator and the optical fields are [40]

$$
\begin{aligned}
\ddot{x}= & -\omega_{o}^{2}\left(x-x_{\mathrm{pt}}\right) \\
& -\frac{\hbar k U_{o}}{m} \sum_{j} a_{j}^{\dagger} a_{j} \sin \left[2\left(k x+\phi_{j}\right)\right]-\gamma_{g} \dot{x}+\frac{\zeta}{m}, \\
\dot{a}_{j}= & -\left(\kappa-i \Delta_{o}^{j}\right) a_{j}+i U_{o} a_{j} \cos ^{2}\left(k x+\phi_{j}\right) \\
& +\sqrt{2 \kappa_{\mathrm{in}}} \alpha_{\mathrm{in}, j}+v_{j},
\end{aligned}
$$

where $j=p, t$ indicate the probe and trap fields, respectively. In Eq. (1), $\omega_{o}$ is the Paul trap secular frequency, $x_{\mathrm{pt}}$ its origin, $\gamma_{g}$ the gas damping, $\Delta_{o}^{j}$ is the empty cavity detuning, $\kappa=\kappa_{\text {in }}+\kappa_{\text {loss }}$ is the total cavity half linewidth, $\alpha_{\text {in, } j}$ are the driving amplitudes, $k$ is the trapping field wave number, and $v_{i}=\sqrt{2 \kappa_{\text {in }}} a_{\text {in }, j}+\sqrt{2 \kappa_{\text {loss }}} a_{\text {loss }, j}$ is a weighted sum of all vacuum operators. Field fluctuations are uncorrelated with the only nonvanishing correlation function given by $\left\langle a_{i}(t) a_{j}^{\dagger}\left(t^{\prime}\right)\right\rangle=\delta\left(t-t^{\prime}\right) \delta_{i j}$ and $\zeta$ is a Brownian stochastic force that arises from background gas collisions and with a correlation function given by $\left\langle\zeta(t) \zeta\left(t^{\prime}\right)\right\rangle=2 k_{B} T_{\text {bath }} m \gamma_{g} \delta(t-$ $\left.t^{\prime}\right)=S_{\mathrm{th}} \delta\left(t-t^{\prime}\right)$, where $T_{\text {bath }}$ is the temperature of the background gas.

The particle dynamics given by Eq. (1) can be radically different depending on the chosen parameters. This occurs because there are three competing contributions to the trapping potential and a strong nonlinearity introduced by the cavity standing waves. For example, if the particle mean position $x_{o}$ is significantly different from the Paul trap center $x_{\mathrm{pt}}$, one obtains a time-dependent optomechanical coupling [25,41]. If the intracavity field amplitudes of the trapping and probe beams are comparable, the particle mean position is pulled from the trapping beam antinode. In this way one obtains a dominant linear coupling for both beams [38], recovering the most common optomechanical interaction, which can be exploited for standard cavity cooling. Here, we consider a scenario where the probe power is significantly weaker than the trap beam, i.e., $\alpha_{\mathrm{in}, p} \ll \alpha_{\mathrm{in}, t}$, so that the particle mean position remains in an antinode of the trapping beam which leads to a vanishingly small linear coupling. Additionally, we consider $x_{o} \simeq x_{\mathrm{pt}}$, so that the Paul trap potential can be neglected, and that $x_{o}$ is not at the cavity center. Under these conditions the particle dynamics are dominated by the trapping field. The probe field will have a linear coupling which can be exploited to detect the particle motion. Indeed, since the two fields have different frequencies there will be a phase difference $\phi_{p}=\pi / 2+\pi x_{o} / L_{\mathrm{cav}}$ [38], where the origin has been chosen at the cavity center. To gain a clearer understanding of the oscillator dynamics it is convenient to approximate Eq. (1) and eliminate the cavity dynamics [42], which leads to an approximate equation of motion for the particle in the following form,

$$
\ddot{x}=-\Omega_{m}^{2} x\left(1+\epsilon_{D} x^{2}\right)-\left(\gamma_{g}-\Omega_{m}^{2} \gamma_{\mathrm{nl}} x^{2}\right) \dot{x}+\frac{\zeta}{m},
$$

where $x$ now represents the fluctuations around the steady state position $x_{o}, \Omega_{m}$ is the optical trap frequency, and where we have introduced two additional terms: an elastic Duffing nonlinearity $\epsilon_{D}$ and a van der Pol nonlinear damping $\gamma_{\mathrm{nl}}$. The latter, for $\gamma_{\mathrm{nl}}<0$, corresponds to a dissipation process that becomes more efficient for large amplitude oscillations. 
In Eq. (2), we neglect the effect of vacuum fluctuations which must be included to model the dynamics in the quantum regime [30]. Equation (2) can be derived from Eq. (1) by neglecting the effect of the probe beam, assuming that $\Omega_{m} / \kappa \ll 1$, and by following the method described in Ref. [42], we obtain

$$
\epsilon_{D}=\frac{2 G_{2}}{\kappa} \frac{\delta}{\left(1+\delta^{2}\right)}, \quad \gamma_{\mathrm{nl}}=\frac{8 G_{2}}{\kappa^{2}} \frac{\delta}{\left(1+\delta^{2}\right)^{2}},
$$

where $\delta=\Delta / \kappa$ is the normalized cavity detuning of the trapping beam which is referred to as the particle shifted cavity resonance, i.e., $\Delta=\Delta_{o}^{t}-\Delta\left(x_{o}\right) ; G_{2}=k^{2} U_{o}$ is the quadratic coupling, $\Omega_{m}^{2}=\frac{2 \hbar G_{2}}{m}\left|\alpha_{t, s}\right|^{2}$, where $\alpha_{t, s}$ is the steady state intracavity field amplitude. Equations (3) are valid under the additional condition $G_{2}\left\langle x^{2}\right\rangle \ll \kappa$ which is always satisfied in our experiment. As for the linear coupling, for a red detuned $(\delta<0)$ trapping beam the optical potential is softened and dissipation is increased while the opposite happens for a blue detuned beam, which can result in a dynamical instability. Interestingly, both nonlinear coefficients are power independent, which is a characteristic of levitation since there is no intrinsic elastic potential as in other optomechanical platforms.

It is quite convenient, at this point, to move to a reference frame rotating at $\Omega_{m}$ and to write the equation of motion for the amplitude $R(t)$ and phase $\varphi(t)$ of the oscillator. By performing deterministic and stochastic averaging [43-45], valid in the high $Q$ limit, one obtains two first-order differential equations for the amplitude $R$ and phase $\varphi$ given by

$$
\begin{aligned}
& \dot{R}=-\frac{\gamma_{g}}{2} R+\frac{\Omega_{m}^{2} \gamma_{\mathrm{nl}}}{8} R^{3}+\frac{S_{\mathrm{th}}}{4 m^{2} \Omega_{m}^{2} R}+\xi=-\frac{d \mathcal{V}(R)}{d R}+\xi \\
& \dot{\varphi}=\frac{3 \Omega_{m}}{8} \epsilon_{D} R^{2}+\frac{1}{R} \chi
\end{aligned}
$$

Here, $\xi$ and $\chi$ are two uncorrelated stochastic variables with a correlation function $\left\langle\xi(t) \xi\left(t^{\prime}\right)\right\rangle=\left\langle\chi(t) \chi\left(t^{\prime}\right)\right\rangle=$ $\left(S_{\mathrm{th}} / 2 m^{2} \Omega_{m}^{2}\right) \delta\left(t-t^{\prime}\right)$ and where we introduced the potential $\mathcal{V}(R)$. Equations (4) allow us to highlight two key aspects. The Duffing term only affects the evolution of the phase and has no effect on the energy of the oscillator. The evolution of the amplitude is phase independent, which allows us to write a simple one-dimensional Fokker-Planck (FP) equation for the evolution of the probability density function (PDF) of $R$ whose steady state solution is well known and given by $P_{\infty}(R)=\mathcal{N} \exp \left[-4 m^{2} \Omega_{m}^{2} \mathcal{V}(R) / S_{\mathrm{th}}\right]$ [46]. This reduces to the familiar Rayleigh distribution in the limit of vanishing nonlinear damping. Expressing the steady state solution of the FP equation in terms of energy $E=m \Omega_{m}^{2} R^{2} / 2$ rather than amplitude gives

$$
P_{\infty}(E)=\frac{\mathcal{N}}{m \Omega_{m}^{2}} \exp \left[-\frac{E}{k_{B} T_{\text {bath }}}\left(1+\frac{\gamma_{n l}}{4 m \gamma_{g}} E\right)\right],
$$

where $\mathcal{N}$ is a normalization constant such that $\int_{0}^{\infty} P_{\infty}(E) d E=1$. Since the energy distribution is known, all the relevant dynamical parameters can be obtained. For a vanishing nonlinear damping Eq. (5) becomes the usual Boltzmann-Gibbs distribution.

It is important to notice that the coupled dynamics described here are formally equivalent to active parametric

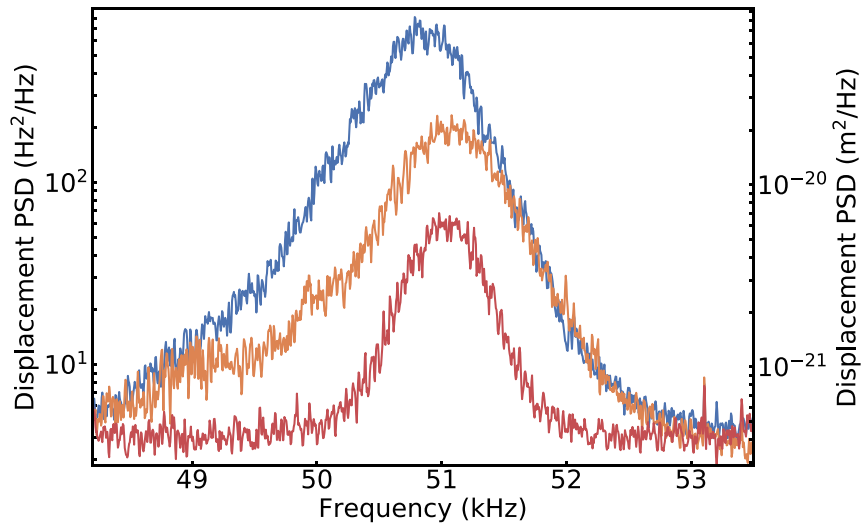

FIG. 2. Calibrated power spectral density (PSD) of the frequency fluctuations induced by the particle motion in the PDH error signal at different pressures. The blue, orange, and red PSDs show the particle displacement at pressures $1.2 \times 10^{-2}, 8.6 \times 10^{-4}$ and $5.4 \times 10^{-6} \mathrm{mbar}$, respectively

feedback [29]. Indeed, Eq. (5) describes the steady state energy distribution of both processes. This occurs as in both cases the oscillator dynamic is modified by an optical force proportional to $x^{2} \dot{x}$, i.e., a van der Pol nonlinear damping. As such, quadratic coupling can also be viewed as a passive parametric feedback. Furthermore, Eq. (5) represents the classical limit of two-phonon cooling in the quantum regime [47].

Here, we present data obtained with a probe and trap beam input power of 2.9 and $830 \mu \mathrm{W}$, respectively. The probe is locked near resonance while the trap beam has a nominal red detuning of $\Delta / 2 \pi \simeq-100 \mathrm{kHz}$, chosen to maximize $\gamma_{\mathrm{nl}}$ in Eq. (3). The particle motion is monitored through the PDH error signal since its linear coupling enables a direct measurement of the mechanical motion. Spectra at different pressures are shown in Fig. 2. It is clear that the oscillator resonance at $\Omega_{m} / 2 \pi \simeq 51 \mathrm{kHz}$ does not converge to a Lorentzian-like peak, as it should when the pressure is reduced, but converges to a Gaussian peak. This broadening is due to low-frequency intensity fluctuations and by cross coupling with the motion in the transverse directions. These effects limit the amount of information that can be extracted from the PDH spectra shown in this figure. However, both are eliminated from the evolution of the amplitude $R$ when moving to the rotating frame as is the case for the Duffing nonlinearity which highlights the usefulness of the method.

We obtain a time series for the amplitude quadrature $R(t)$ from the PDH signal in postprocessing (see Refs. [30,48] for details). Its analysis in the time domain gives us the oscillator energy distribution which we compare with the model predictions, while its spectral analysis allows us a direct estimation of the effective damping.

The experimental energy distribution is shown in Figs. 3(a) and 3(b) at two different pressures where the distributions are expressed in units of $k_{B}$, i.e., temperature, for a more intuitive reading. At the higher pressure of $P_{g}=8.6 \times 10^{-2}$ mbar [Fig. 3(a)], the deviation from a thermal exponential distribution is immediately recognizable, and indeed the nonlinear damping is much more efficient in suppressing large amplitude fluctuations. At the lower pressure of 

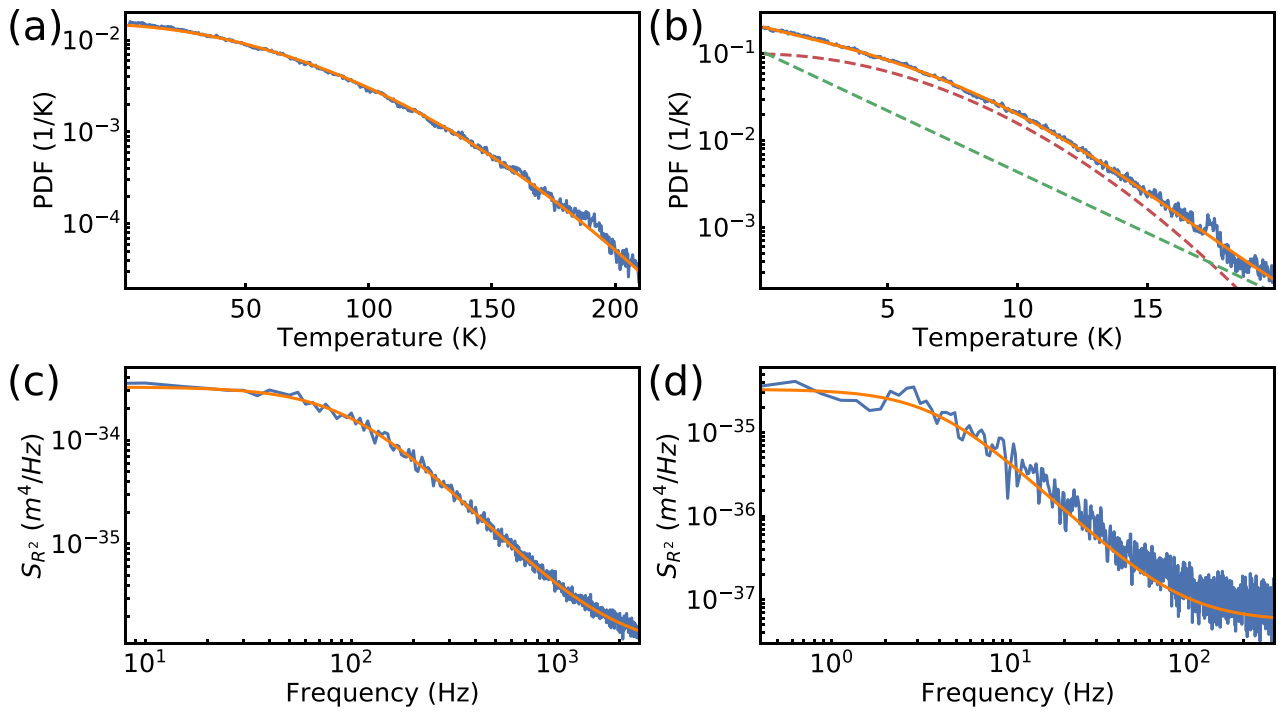

FIG. 3. (a) and (b) Energy distribution (blue), expressed in units of $k_{B}$, for the motion along the $x$ axis (cavity axis) along with a fit (orange) following Eq. (5) for different pressures $P_{g}=8.6 \times 10^{-2}$ and $1.6 \times 10^{-4} \mathrm{mbar}$, respectively. In (b) the contribution of the detection noise is included; the corresponding marginal distributions for the motion (red dashed) and noise (green dashed) are also shown. (c) and (d) show the PSD of $R^{2}$ corresponding to the distributions in (a) and (b), respectively; fits (orange) of the $R^{2}$ spectra allow a direct estimation of the energy autocorrelation time constant (see main text). (e) Effective temperature as a function of fitted parametric gain $\eta=\gamma_{\mathrm{nl}} / \gamma_{g}$ along with $a$ priori analytical estimation [30] (orange line). The shaded region indicates the uncertainty of the theoretical curves due to experimental uncertainty; blue and green data points correspond to localization on slightly different optical wells. The red square marks the data point corresponding to (a) and (c), the black square to (b) and (d).

$P_{g}=1.6 \times 10^{-4}$ mbar [Fig. 3(b)] this behavior is initially less evident. As the motion becomes colder, the impact of the detection noise becomes more relevant and needs to be taken into account. Assuming the noise floor is white and uncorrelated with the motion, its distribution is again exponential. We fit the experimental data taking into account both processes. The oscillator energy PDF is then recovered by taking the marginal distribution. As the intrinsic gas damping cannot be measured independently, and our pressure gauges have a rather low accuracy $(\sim 30 \%)$, we use as a fitting parameter the ratio $\eta=\gamma_{\mathrm{nl}} / \gamma_{g}$, which can be interpreted as a parametric gain.

We show in Fig. 3(e) the experimental effective temperature $T_{\text {eff }}$ as a function of $\eta$, along with an a priori analytical estimation [30] which considers the nonlinear damping to be $\gamma_{\mathrm{nl}}=-2.5 \times 10^{7} \mathrm{~s} / \mathrm{m}^{2}$, in good agreement with experimental results. The final temperature can be estimated in two ways: from the area of the peak in the probe PSD (Fig. 2), as is typical, and from the expectation value of the fitted distribution. The consistency of these two estimates which are well within the experimental uncertainty, as well as the agreement with the analytical expectation, demonstrate that even at the lowest pressure the quadratic coupling is dominating the dynamics.

When comparing theory and experimental results in Fig. 3(e) and later in Fig. 4, we also consider how the analytical calculations vary according to experimental uncertainty on the relevant parameters. This is shown as a shaded region which is calculated by performing a global maximization (minimization) to find the upper (lower) bound when all possible combinations of experimental uncertainties are considered at the $1 \sigma$ level. The parameters included are pressure, de- tuning, calibration, and cavity decay rate. Pressure gives the largest contribution, the decay rate the smallest.

Another clear signature of the nonlinear damping is the dependence on pressure of the effective temperature. An approximate expression can be obtained from Eq. (5); in the low-pressure limit, we have $T_{\text {eff }}=\left(4 m T_{\text {bath }} / \pi k_{B} \eta\right)^{1 / 2}$. Since $\eta$ grows inversely proportionally to the pressure, the effective temperature decreases proportionally to the square root of the pressure. This is shown in Fig. 4 where the experimental observation is compared with an analytical estimation. A direct consequence is that the effective total damping $\gamma_{\text {eff }}$ must have a similar behavior; here, $\gamma_{\text {eff }}$ is to be interpreted as the equivalent viscous friction that would lead to the effective temperature $T_{\text {eff }}$, i.e., $T_{\text {eff }}=T_{\text {bath }} \gamma_{g} / \gamma_{\text {eff. }}$. In the low-pressure limit we have $\gamma_{\text {eff }}=\gamma_{g}\left(\pi k_{B} T_{\text {bath }} \eta / 4 m\right)^{1 / 2}$. This implies a perfect correlation between $T_{\text {eff }}$ and $\gamma_{\text {eff }}$ as the pressure is reduced since both depend on the square root of the pressure.

A direct estimation of the effective damping can be obtained even in the presence of the Duffing term and of the broadening of the spectral peak, evident in Fig. 2. By looking at the PSD of $R^{2}(t)$ it is possible to obtain information on the energy autocorrelation time constant. PSDs at two different pressures are shown in Fig. 3. Although possible for a thermal oscillator $[48,49]$, calculating an analytical expression for the $R^{2} \mathrm{PSD}$, when the dynamic is dominated by a nonlinear damping, is not trivial. However, it can be shown that modeling the PSD as $S_{R^{2} R^{2}}(\omega)=16 \gamma_{R} a_{o}^{2} /\left(\omega^{2}+\gamma_{R}^{2}\right)$ then $\gamma_{R}$ allows us to calculate the effective damping as $\gamma_{\text {eff }}=\gamma_{R} \sigma_{E}^{2} /\langle E\rangle^{2}$, where $\langle E\rangle^{2}$ and $\sigma_{E}^{2}$ are the energy mean and variance, respectively, both of which can be calculated from the fit to the experimental distribution. The effective damping calculated with this 

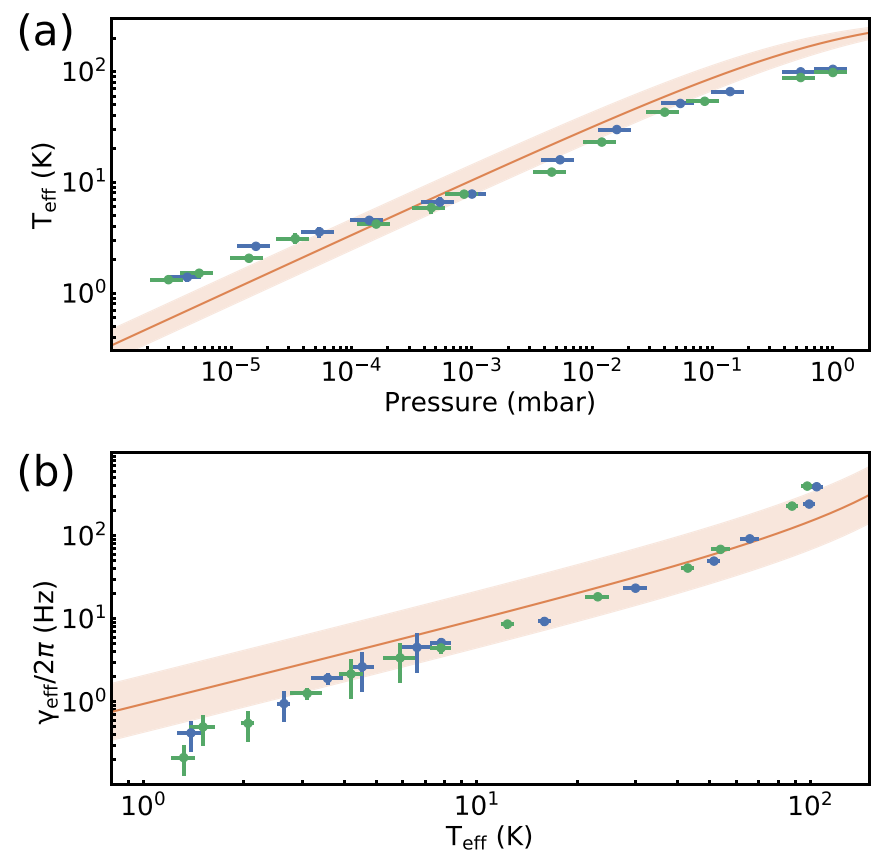

FIG. 4. (a) The effective temperature as a function of pressure. (b) The measured effective damping as a function of $T_{\text {eff }}$. In both panels blue and green data points correspond to localization on slightly different optical wells; also shown is an analytical estimation (orange line) with the shaded region indicating the uncertainty of the theoretical curves.

method, plotted as a function of $T_{\text {eff }}$, is shown in Fig. 4(b), demonstrating the expected good correlation.

In conclusion, we have demonstrated strong quadratic cooling of the c.m. motion of a nanoparticle optically trapped in a cavity field. A comparison of the experimental results shows good agreement with an analytical a priori model. This type of passive parametric feedback cooling is analogous to the active feedback cooling in levitated optical tweezers and indeed comparable temperatures are obtained here. The major difference between the two methods is that the cavity automatically applies feedback whereas conventionally detection and electronic feedback are required. In both cases, the cooling rate decreases as the particle is cooled to the bottom of the optical potential. We have also shown that a highly nonthermal state is produced. The quadratic cooling power demonstrated here can be improved by more than two orders of magnitude by increasing the cavity finesse within realistic values. However, reaching the quantum regime with just this protocol remains challenging and more complex ones are likely to be necessary. As we can dynamically manipulate the coupling between quadratic and linear by simply controlling the power ratio between the probe and trapping field, the particle could be initialized close to the quantum ground state [2] through a linear coupling and then adiabatically changed to a quadratic coupling where nonclassical states [47] and nonequilibrium states could be created. This dynamically tunable cavity system may also be useful for exploring nanoscale thermodynamics, allowing the measurement of the relative entropy change for testing nonequilbrium thermodynamics and fluctuation theorems [29,50-52].

The authors would like to acknowledge useful discussions with M. Toroš. The authors acknowledge funding from the EPSRC Grant No. EP/N031105/1. N.P.B. acknowledges funding from the EPSRC Grant No. EP/L015242/1. A.P. has received funding from the European Union's Horizon 2020 research and innovation programme under the Marie Sklodowska-Curie Grant Agreement No. 749709.
[1] J. Chan, T. P. M. Alegre, A. H. Safavi-Naeini, J. T. Hill, A. Krause, S. Gröblacher, M. Aspelmeyer, and O. Painter, Laser cooling of a nanomechanical oscillator into its quantum ground state, Nature (London) 478, 89 (2011).

[2] U. Delić, M. Reisenbauer, K. Dare, D. Grass, V. Vuletić, N. Kiesel, and M. Aspelmeyer, Cooling of a levitated nanoparticle to the motional quantum ground state, Science 367, 892 (2020).

[3] E. E. Wollman, C. U. Lei, A. J. Weinstein, J. Suh, A. Kronwald, F. Marquardt, A. A. Clerk, and K. C. Schwab, Quantum squeezing of motion in a mechanical resonator, Science 349, 952 (2015).

[4] C. F. Ockeloen-Korppi, E. Damskägg, J.-M. Pirkkalainen, M. Asjad, A. A. Clerk, F. Massel, M. J. Woolley, and M. A. Sillanpää, Stabilized entanglement of massive mechanical oscillators, Nature (London) 556, 478 (2018).

[5] R. Riedinger, A. Wallucks, I. Marinković, C. Löschnauer, M. Aspelmeyer, S. Hong, and S. Gröblacher, Remote quantum entanglement between two micromechanical oscillators, Nature (London) 556, 473 (2018).

[6] P. F. Barker and M. N. Shneider, Cavity cooling of an optically trapped nanoparticle, Phys. Rev. A 81, 023826 (2010).

[7] D. E. Chang, C. A. Regal, S. B. Papp, D. J. Wilson, J. Ye, O. Painter, H. J. Kimble, and P. Zoller, Cavity opto-mechanics using an optically levitated nanosphere, Proc. Natl. Acad. Sci. USA 107, 1005 (2010).

[8] O. Romero-Isart, M. L. Juan, R. Quidant, and J. I. Cirac, Toward quantum superposition of living organisms, New J. Phys. 12, 033015 (2010).

[9] Y. Arita, M. Mazilu, and K. Dholakia, Laser-induced rotation and cooling of a trapped microgyroscope in vacuum, Nat. Commun. 4, 2374 (2013).

[10] T. Li, S. Kheifets, D. Medellin, and M. G. Raizen, Measurement of the instantaneous velocity of a Brownian particle, Science 328, 1673 (2010).

[11] J. Gieseler and J. Millen, Levitated nanoparticles for microscopic thermodynamics-a review, Entropy 20, 326 (2018).

[12] W.-J. Gu, Z. Yi, L.-H. Sun, and D. H. Xu, Mechanical cooling in single-photon optomechanics with quadratic nonlinearity, Phys. Rev. A 92, 023811 (2015).

[13] H. Shi and M. Bhattacharya, Quantum mechanical study of a generic quadratically coupled optomechanical system, Phys. Rev. A 87, 043829 (2013).

[14] Z. J. Deng, Y. Li, M. Gao, and C. W. Wu, Performance of a cooling method by quadratic coupling at high temperatures, Phys. Rev. A 85, 025804 (2012). 
[15] A. Xuereb and M. Paternostro, Selectable linear or quadratic coupling in an optomechanical system, Phys. Rev. A 87, 023830 (2013).

[16] J. D. P. Machado, R. J. Slooter, and Y. M. Blanter, Quantum signatures in quadratic optomechanics, Phys. Rev. A 99, 053801 (2019).

[17] H. Tan, F. Bariani, G. Li, and P. Meystre, Generation of macroscopic quantum superpositions of optomechanical oscillators by dissipation, Phys. Rev. A 88, 023817 (2013).

[18] T. V. Gevorgyan, A. R. Shahinyan, and G. Y. Kryuchkyan, Generation of Fock states and qubits in periodically pulsed nonlinear oscillators, Phys. Rev. A 85, 053802 (2012).

[19] S. Rips, M. Kiffner, I. Wilson-Rae, and M. J. Hartmann, Steadystate negative Wigner functions of nonlinear nanomechanical oscillators, New J. Phys. 14, 023042 (2012).

[20] J. C. Sankey, C. Yang, B. M. Zwickl, A. M. Jayich, and J. G. E. Harris, Strong and tunable nonlinear optomechanical coupling in a low-loss system, Nat. Phys. 6, 707 (2010).

[21] M. Karuza, M. Galassi, C. Biancofiore, C. Molinelli, R. Natali, P. Tombesi, G. D. Giuseppe, and D. Vitali, Tunable linear and quadratic optomechanical coupling for a tilted membrane within an optical cavity: Theory and experiment, J. Opt. 15, 025704 (2012).

[22] T. K. Paraïso, M. Kalaee, L. Zang, H. Pfeifer, F. Marquardt, and O. Painter, Position-Squared Coupling in a Tunable Photonic Crystal Optomechanical Cavity, Phys. Rev. X 5, 041024 (2015).

[23] H. Kaviani, C. Healey, M. Wu, R. Ghobadi, A. Hryciw, and P. E. Barclay, Nonlinear optomechanical paddle nanocavities, Optica 2, 271 (2015).

[24] C. Healey, H. Kaviani, M. Wu, B. Khanaliloo, M. Mitchell, A. C. Hryciw, and P. E. Barclay, Design and experimental demonstration of optomechanical paddle nanocavities, Appl. Phys. Lett. 107, 231107 (2015).

[25] P. Z. G. Fonseca, E. B. Aranas, J. Millen, T. S. Monteiro, and P. F. Barker, Nonlinear Dynamics and Strong Cavity Cooling of Levitated Nanoparticles, Phys. Rev. Lett. 117, 173602 (2016).

[26] U. Delić, M. Reisenbauer, D. Grass, N. Kiesel, V. Vuletic, and M. Aspelmeyer, Cavity Cooling of a Levitated Nanosphere by Coherent Scattering, Phys. Rev. Lett. 122, 123602 (2019).

[27] U. Delić, D. Grass, M. Reisenbauer, T. Damm, M. Weitz, N. Kiesel, and M. Aspelmeyer, Levitated cavity optomechanics in high vacuum, Quantum Sci. Technol. 5, 025006 (2020).

[28] J. Gieseler, B. Deutsch, R. Quidant, and L. Novotny, Subkelvin Parametric Feedback Cooling of a Laser-Trapped Nanoparticle, Phys. Rev. Lett. 109, 103603 (2012).

[29] J. Gieseler, R. Quidant, C. Dellago, and L. Novotny, Dynamic relaxation of a levitated nanoparticle from a non-equilibrium steady state, Nat. Nanotechnol. 9, 358 (2014).

[30] See Supplemental Material at http://link.aps.org/supplemental/ 10.1103/PhysRevResearch.3.L032022 for a complete description of the model and additional details on the experimental setup and calibrations.

[31] N. P. Bullier, A. Pontin, and P. F. Barker, Characterisation of a charged particle levitated nano-oscillator, J. Phys. D 53, 175302 (2020).

[32] A. C. Pflanzer, O. Romero-Isart, and J. I. Cirac, Masterequation approach to optomechanics with arbitrary dielectrics, Phys. Rev. A 86, 013802 (2012).

[33] F. Ricci, M. T. Cuairan, G. P. Conangla, A. W. Schell, and R. Quidant, Accurate mass measurement of a levitated nanome- chanical resonator for precision force-sensing, Nano Lett. 19, 6711 (2019).

[34] F. García-Santamaría, H. Míguez, M. Ibisate, F. Meseguer, and C. López, Refractive index properties of calcined silica submicrometer spheres, Langmuir 18, 1942 (2002).

[35] J. Millen, T. Deesuwan, P. Barker, and J. Anders, Nanoscale temperature measurements using non-equilibrium Brownian dynamics of a levitated nanosphere, Nat. Nanotechnol. 9, 425 (2014).

[36] E. Hebestreit, R. Reimann, M. Frimmer, and L. Novotny, Measuring the internal temperature of a levitated nanoparticle in high vacuum, Phys. Rev. A 97, 043803 (2018).

[37] N. P. Bullier, A. Pontin, and P. F. Barker, Super-resolution imaging of a low frequency levitated oscillator, Rev. Sci. Instrum. 90, 093201 (2019).

[38] N. Kiesel, F. Blaser, U. Delić, D. Grass, R. Kaltenbaek, and M. Aspelmeyer, Cavity cooling of an optically levitated submicron particle, Proc. Natl. Acad. Sci. USA 110, 14180 (2013).

[39] J. Millen, P. Z. G. Fonseca, T. Mavrogordatos, T. S. Monteiro, and P. F. Barker, Cavity Cooling a Single Charged Levitated Nanosphere, Phys. Rev. Lett. 114, 123602 (2015).

[40] T. S. Monteiro, J. Millen, G. A. T. Pender, F. Marquardt, D. Chang, and P. F. Barker, Dynamics of levitated nanospheres: Towards the strong coupling regime, New J. Phys. 15, 015001 (2013).

[41] E. B. Aranas, P. Z. G. Fonseca, P. F. Barker, and T. S. Monteiro, Split-sideband spectroscopy in slowly modulated optomechanics, New J. Phys. 18, 113021 (2016).

[42] C. Jiang, Y. Cui, and G. Chen, Dynamics of an optomechanical system with quadratic coupling: Effect of first order correction to adiabatic elimination, Sci. Rep. 6, 35583 (2016).

[43] R. Stratonovich, Topics in the Theory of Random Noise (Gordon and Breach, New York, 1967), Vol. 2.

[44] J. Roberts and P. Spanos, Stochastic averaging: An approximate method of solving random vibration problems, Int. J. Non Linear Mech. 21, 111 (1986).

[45] E. Boujo and N. Noiray, Robust identification of harmonic oscillator parameters using the adjoint Fokker-Planck equation, Proc. R. Soc. A 473, 20160894 (2017).

[46] J. Bendat and A. Piersol, Random Data: Analysis and Measurement Procedures, 4th ed. (Wiley, Hoboken, NJ, 2011).

[47] A. Nunnenkamp, K. Børkje, J. G. E. Harris, and S. M. Girvin, Cooling and squeezing via quadratic optomechanical coupling, Phys. Rev. A 82, 021806(R) (2010).

[48] A. Pontin, N. P. Bullier, M. Toroš, and P. F. Barker, Ultranarrow-linewidth levitated nano-oscillator for testing dissipative wave-function collapse, Phys. Rev. Research 2, 023349 (2020).

[49] D. Zheng, Y. Leng, X. Kong et al., Room temperature test of the continuous spontaneous localization model using a levitated micro-oscillator, Phys. Rev. Research 2, 013057 (2020).

[50] D. J. Evans, E. G. D. Cohen, and G. P. Morriss, Probability of Second Law Violations in Shearing Steady States, Phys. Rev. Lett. 71, 2401 (1993).

[51] D. J. Evans and D. J. Searles, Equilibrium microstates which generate second law violating steady states, Phys. Rev. E 50, 1645 (1994).

[52] G. Gallavotti and E. G. D. Cohen, Dynamical ensembles in stationary states, J. Stat. Phys. 80, 931 (1995). 